\title{
VARIATIONS ON A THEOREM OF COWLING AND PRICE WITH APPLICATIONS TO NILPOTENT LIE GROUPS
}

\author{
S. PARUI ${ }^{\square}$ and S. THANGAVELU
}

(Received 16 July 2004; revised 22 June 2005)

Communicated by A. H. Dooley

\begin{abstract}
In this paper we prove a new version of the Cowling-Price theorem for Fourier transforms on $\mathbb{R}^{n}$. Using this we formulate and prove an uncertainty principle for operators. This leads to an analogue of the Cowling-Price theorem for nilpotent Lie groups. We also prove an exact analogue of the Cowling-Price theorem for the Heisenberg group.
\end{abstract}

2000 Mathematics subject classification: primary 43A30.

Keywords and phrases: Weyl transform, nilpotent Lie groups, heat kernel, Hermite and special Hermite functions.

\section{Introduction}

Consider functions $f$ on $\mathbb{R}^{n}$ that satisfy estimates of the form

$$
|f(x)| \leq C e^{-a|x|^{2}}, \quad|\hat{f}(\xi)| \leq C e^{-b|\xi|^{2}}
$$

for some $a, b>0$ where $\hat{f}$ is the Fourier transform of $f$ defined by

$$
\hat{f}(\xi)=(2 \pi)^{-n / 2} \int_{\mathbb{R}^{n}} f(x) e^{-i x \xi} d x .
$$

A classical theorem of Hardy [6] proved way back in 1933 states that for nontrivial $f$, the product $a b$ is at most $1 / 4$ and the maximum value is attained precisely when $f(x)=c e^{-a|x|^{2}}$. Since $a b>1 / 4$ implies $f=0$, the result of Hardy is an example of an uncertainty principle for the Fourier transform. The case $a b=1 / 4$ is considered a characterisation of the Gaussian.

(C) 2007 Australian Mathematical Society $1446-7887 / 07 \$ A 2.00+0.00$ 
In 1983, Cowling and Price [5] obtained a generalization of Hardy's theorem. Let us define $\phi_{a}(x)=e^{-a|x|^{2}}$. Then we have the following result.

THEOREM 1.1 (Cowling-Price). For $1 \leq p, q \leq \infty$, let $f \phi_{a}^{-1} \in L^{p}\left(\mathbb{R}^{n}\right)$ and $\hat{f} \phi_{b}^{-1} \in L^{q}\left(\mathbb{R}^{n}\right)$. Then $f=0$ whenever $a b>1 / 4$.

The case $p=q=\infty$ in the above theorem is Hardy's theorem with $a b>1 / 4$. The above result is true even if $a b=1 / 4$ under the added assumption that $\min (p, q)<\infty$. A further generalization of the Cowling-Price theorem for the case $a b=1 / 4$ has been recently obtained by Bonami et al. [4].

Analogues of Hardy and Cowling-Price theorems have received considerable attention during the last decade, see the monograph [15] and the references there. In this paper, we are mainly concerned with the Cowling-Price theorem for the group Fourier transform on nilpotent Lie groups. Such a theorem can be proved for the simplest case of the Heisenberg group.

Let $H^{n}$ be the $(2 n+1)$-dimensional Heisenberg group and let $\hat{f}(\lambda), \lambda \in \mathbb{R} \backslash\{0\}$ be the group Fourier transform of a function $f$ on $H^{n}$. The role of the Gaussian $\phi_{a}$ will be played by the heat kernel $q_{a}(z, t)$ associated with the sublaplacian on $H^{n}$. Let $H(\lambda)=-\Delta+\lambda^{2}|x|^{2}$ be the scaled Hermite operator. Then we have the following result.

THEOREM 1.2. Let $f$ be a function on $H^{n}$ that satisfies $f q_{a}^{-1} \in L^{p}\left(H^{n}\right), 1 \leq p \leq \infty$ and let $\hat{f}(\lambda) e^{b H(\lambda)}$ be a bounded operator in $L^{2}\left(\mathbb{R}^{n}\right)$ for every $\lambda \in \mathbb{B} \backslash\{0\}$. Then $f=0$ whenever $a<b$.

The case $p=\infty$ is Hardy's theorem for the Heisenberg group, which can be found in [14]. We are interested in finding an analogue of the above result for stratified nilpotent groups. An examination of the proof of the above theorem, given in Section 3, reveals that we need explicit formula for the heat kernel and good estimates. Due to the lack of such information in the general case, we look for alternative versions of the Cowling-Price theorem.

Returning to the Euclidean case, consider the Gaussian $\phi_{a}(x)$ for which $\hat{\phi}_{a}=C \phi_{b}$ with $b=1 / 4 a$. In view of the Plancherel theorem, we have

$$
\left\|\partial^{\alpha} \hat{\phi}_{a}\right\|_{2}^{2}=\int_{\mathbb{R}^{n}}\left|x^{\alpha} \phi_{a}(x)\right|^{2} d x=2^{n} \prod_{j=1}^{n} \int_{0}^{\infty} t^{2 \alpha_{j}} e^{-2 a t^{2}} d t,
$$

which gives the estimate

$$
\left\|\partial^{\alpha} \hat{\phi}_{a}\right\|_{2}^{2}=C \prod_{j=1}^{n} \Gamma\left(\alpha_{j}+\frac{1}{2}\right)(2 a)^{-|\alpha|} \leq C \alpha !(2 b)^{|\alpha|} .
$$


If a function $f$ satisfies $|f(x)| \leq C \phi_{a}(x)$, then the derivatives of $\hat{f}$ satisfy the estimates

$$
\left\|\partial^{\alpha} \hat{f}\right\|_{2}^{2} \leq C \alpha !(2 a)^{-|\alpha|} .
$$

Replacing the pointwise estimate $|f(x)| \leq C \phi_{a}(x)$ by the slightly weaker estimates (1.3), we arrive at the following uncertainty principle.

THEOREM 1.3. Let $f$ be a function on $\mathbb{R}^{n}$ such that $|\hat{f}(\xi)| \leq C e^{-b|\xi|^{2}}$, and for every $\alpha \in \mathbb{N}^{n}$, we have $\left\|\partial^{\alpha} \hat{f}\right\|_{2}^{2} \leq C \alpha !(2 a)^{-|\alpha|}$. Then $f=0$ whenever $a b>1 / 4$. When $a b=1 / 4, \hat{f}(\xi)=\phi(\xi) e^{-b|\xi|^{2}}$, where $\phi$ is an entire function on $\mathbb{C}^{n}$, but its restriction on $\mathbb{R}^{n}$ is a bounded function.

We remark that Theorem 1.3 (case $a b>1 / 4$ ) is equivalent to Theorem 1.1 (case $p=2, q=\infty$ ). Thus Theorem 1.3 is another version of the Cowling-Price theorem. Stated in this form it has natural extension to the case of nilpotent Lie groups.

The group Fourier transform on a nilpotent Lie group $G$ is operator valued. Given an irreducible unitary representation $\pi$ of $G$ and a function $f$ on $G$, the operator $\hat{f}(\pi)=\pi(f)$ is realised on $L^{2}\left(\mathbb{R}^{n}\right)$ for a suitable $n$. In order to formulate an analogue of Theorem 1.3, we need such a result for operators.

Given a bounded linear operator $T$ on $L^{2}\left(\mathbb{R}^{n}\right)$, we define certain noncommutative derivations of $T$ by

$$
\delta_{j} T=\left[A_{j}, T\right], \quad \overline{\delta_{j}} T=\left[T, A_{j}^{*}\right],
$$

where $[T, S]=T S-S T$ is the commutator and $A_{j}=\partial / \partial \xi_{j}+\xi_{j}, A_{j}^{*}=-\partial / \partial \xi_{j}+\xi_{j}$ are the annihilation and creation operators. The above derivations were introduced by Mauceri [8] and the second author has used them on several occasions, see $[12,15]$. For multiindices $\alpha, \beta$, we define $\delta^{\alpha} T$ and $\bar{\delta}^{\beta} T$ iteratively. Let $H=-\Delta+|x|^{2}$ be the Hermite operator on $\mathbb{R}^{n}$ which generates the semigroup $e^{-t H}, t>0$. We denote by $\mathscr{S}_{q}(q>0)$ the set of all linear operators $T$ on $L^{2}\left(\mathbb{R}^{n}\right)$ such that $\operatorname{tr}\left(|T|^{q}\right)<\infty$. For $q \geq 1, \mathscr{S}_{q}$ endowed with the norm $\|T\|_{q}:=\left(\operatorname{tr}\left(|T|^{q}\right)\right)^{1 / q}$ is a complete subalgebra of the set of all bounded operators on $L^{2}\left(\mathbb{R}^{n}\right)$. In particular, for $q=2, \mathscr{S}_{2}$ is the Hilbert space of Hilbert-Schmidt operators on $L^{2}\left(\mathbb{R}^{n}\right)$ equipped with the inner product $(T, S)=\operatorname{tr}\left(T S^{*}\right)$. Let $\|T\|_{\mathrm{HS}}$ be the norm of $T$ in this Hilbert space. With these notations we are ready to state our operator analogue of Theorem 1.3.

THEOREM 1.4. Let $T \in \mathscr{S}_{2}$ satisfy the estimates

$$
T^{*} T \leq c e^{-2 b H}, \quad\left\|\delta^{\alpha} \bar{\delta}^{\beta}\left(T^{*} T\right)\right\|_{\mathrm{HS}}^{2} \leq c(\alpha+\beta) ! a^{|\alpha|+|\beta|}
$$

for some $a, b>0$ for all $\alpha, \beta \in N^{n}$. Then $T=0$ whenever $a<2 \tanh 2 b$. 
This theorem is an uncertainty principle for the Weyl transform for the following reason. As is well known every $T \in \mathscr{S}_{2}$ is of the form $W(f)$ for some $f \in L^{2}\left(\mathbb{C}^{n}\right)$; that is to say

$$
T=W(f)=\int_{C^{n}} f(z) \pi(z) d z
$$

where $\pi(z)=\pi_{1}(z, 0), \pi_{1}$ being the Schrödinger representation of $H^{n}$ with parameter $\lambda=1$. The following analogue of Cowling-Price theorem can be proved for the Weyl transform.

THEOREM 1.5. Suppose $f$ on $\mathbb{C}^{n}$ satisfies $f e^{a|z|^{2}} \in L^{p}\left(\mathbb{C}^{n}\right)$ and $W(f) e^{b H} \in \mathscr{S}_{q}$, where $1 \leq p, q \leq \infty$. Then $f=0$ whenever $a \tanh b \geq 1 / 4$ and $\min (p, q)<\infty$. If $p=q=\infty$, then $f=0$ for $a \tanh b>1 / 4$ and $f(z)=C e^{-a|z|^{2}}$ for $a \tanh b=1 / 4$.

As an immediate corollary of Theorem 1.4, we obtain the following theorem for nilpotent Lie groups.

THEOREM 1.6. Let $G$ be a connected, simply connected nilpotent Lie group and let $\Lambda$ be a cross section for the generic coadjoint orbits parametrising the elements of $\hat{G}$ which are relevant for the Plancherel theorem. For each $\lambda \in \Lambda$, let $\pi_{\lambda}$ be the associated element of $\hat{G}$. Let $f \in L^{1} \cap L^{2}(G)$ satisfy the following conditions:

(i) $\pi_{\lambda}(f)^{*} \pi_{\lambda}(f) \leq C e^{-2 b(\lambda) H}$,

(ii) $\left\|\delta^{\alpha} \bar{\delta}^{\beta}\left(\pi_{\lambda}(f)^{*} \pi_{\lambda}(f)\right)\right\|_{\mathrm{HS}}^{2} \leq C(\alpha+\beta) ! a(\lambda)^{|\alpha|+|\beta|}$, where $a(\lambda), b(\lambda)>0$. Then $f=0$ whenever $a(\lambda)<2 \tanh 2 b(\lambda)$ for all $\lambda \in \Lambda$.

For the case of the Heisenberg group, it can be easily checked using the explicit formula for the heat kernel that $\left|f^{\lambda}(z)\right| \leq C q_{a}^{\lambda}(z)$ leads to the estimates

$$
\left\|\delta^{\alpha} \bar{\delta}^{\beta}\left(\hat{f}(\lambda)^{*} \hat{f}(\lambda)\right)\right\|_{\mathrm{HS}}^{2} \leq C(\alpha+\beta) !(a|\lambda|)^{|\alpha|+|\beta|} .
$$

Thus condition (ii) in Theorem 1.6 is a suitable alternative, which compensates for the absence of a good formula for the heat kernel. In the case of the Heisenberg group, we can replace condition (i) by $\hat{f}(\lambda)^{*} \hat{f}(\lambda) \leq C e^{-2 b H(\lambda)}$. Note that $e^{-b H(\lambda)}=\hat{q}_{b}(\lambda)$ and so, it is a natural candidate for measuring the decay of $\hat{f}(\lambda)$. As $H(\lambda)$ is unitarily equivalent to $|\lambda| H$, condition (i) is natural. The same comment applies to the case of all step two groups, as the scaled Hermite operator is related to the sublaplacian even in that case. In the case of general nilpotent groups, there is no canonical way of measuring the decay of $\pi_{\lambda}(f)$. We have used $e^{-b(\lambda) H}$ to measure the decay of the Fourier transform, since we do not have any other choice.

We conclude this section with the following remarks. Different versions of Hardy and Cowling-Price theorems for nilpotent Lie groups have been proved in $[1,2,3,7]$ 
and [10]. In each paper, the conditions are in terms of the Hilbert-Schmidt norm of $\pi_{\lambda}(f)$, and as such are in some sense results for the central variable. This remark is easily justified if one considers functions of the form $f(z, t)=g(z) h(t)$ on the Heisenberg group. The right analogue of Hardy's theorem for $H^{n}$ was proved in [14]. In this paper we try to formulate such an analogue of the Cowling-Price theorem.

\section{Cowling-Price on $\mathbb{R}^{n}$ revisited}

As we mentioned in the introduction, we show that Theorem 1.3 (case $a b>1 / 4$ ) and Theorem 1.1 (case $p=2, q=\infty$ ) are equivalent. However, we first give an independent proof of Theorem 1.3. We start with the following lemma, which allows us to find pointwise estimates on $\partial^{\alpha} \hat{f}$ when we have estimates on $\left\|\partial^{\alpha} \hat{f}\right\|_{2}$.

LEMMA 2.1. Suppose we have $\left\|\partial^{\alpha} \hat{f}\right\|_{2}^{2} \leq C \alpha !(2 a)^{-|\alpha|}$ for every $\alpha \in \mathbb{N}^{n}$. Then we also have $\left|\partial^{\alpha} \hat{f}(\xi)\right|^{2} \leq C \prod_{j=1}^{n}\left(\alpha_{j}+n\right) !(2 a)^{-|\alpha|}$ for every $\alpha \in \mathbb{N}^{n}$.

Proof. In view of the Sobolev embedding theorem,

$$
\left|\partial^{\alpha} \hat{f}(\xi)\right|^{2} \leq C \sum_{|\beta| \leq n}\left\|\partial^{\alpha+\beta} \hat{f}\right\|_{2}^{2}
$$

which gives the estimate

$$
\begin{aligned}
\left|\partial^{\alpha} \hat{f}(\xi)\right|^{2} & \leq C \sum_{|\beta| \leq n}\left\|\partial^{\alpha+\beta} \hat{f}\right\|_{2}^{2} \leq C \sum_{|\beta| \leq n}(2 a)^{-(|\alpha|+|\beta|)}(\alpha+\beta) ! \\
& \leq C \prod_{j=1}^{n}\left(\alpha_{j}+n\right) !(2 a)^{-|\alpha|} .
\end{aligned}
$$

In view of Lemma 2.1, we only need to prove the following version of Theorem 1.3.

THEOREM 2.2. The conclusions of Theorem 1.3 are valid if we replace the estimates on $\left\|\partial^{\alpha} \hat{f}\right\|_{2}$ by $\left|\partial^{\alpha} \hat{f}(\xi)\right|^{2} \leq C \prod_{j=1}^{n}\left(\alpha_{j}+n\right) !(2 a)^{-|\alpha|}$ for every $\alpha \in \mathbb{N}^{n}$.

As an application of the above theorem we have the following corollary.

COROLLARY 2.3. Let $\hat{f}$ be a smooth function such that

$$
\left\|\partial^{\alpha} \hat{f}\right\|_{p}^{2} \leq C \alpha !(2 a)^{-|\alpha|}, \quad|\hat{f}(\xi)| \leq C e^{-b|\xi|^{2}},
$$

where $1 \leq p<\infty$. Then $f=0$ for $a b>1 / 4$. 
PROOF. Choose $t>0$ such that $a>a-t>1 / 4 b$. Consider the function $F_{t}(x)=\hat{f} * p_{t}(x)$, where $p_{t}(x)=(4 \pi t)^{-n / 2} e^{-|x|^{2} / 4 t}$. Now,

$$
\begin{aligned}
\left|\partial^{\alpha} F_{t}(x)\right|^{2} & \leq\left\|p_{t}\right\|_{p^{\prime}}^{2}\left\|\partial^{\alpha} \hat{f}\right\|_{p}^{2} \leq C \alpha !(2 a)^{-|\alpha|}, \\
\left|F_{t}(x)\right| & \leq C e^{-b /(4 b t+1)|x|^{2}} .
\end{aligned}
$$

Therefore, $F_{t}(x)=0$ when $a b /(4 b t+1)>1 / 4$. Hence $f=0$ for $a b>1 / 4$.

We now complete the proof of Theorem 2.2. We first consider the case $a b>1 / 4$. We make use of the following lemma.

LEMMA 2.4. Let $F(\xi)$ be a smooth function on $\mathbb{R}^{n}$ that satisfies

$$
\left|\partial^{\alpha} F(\xi)\right|^{2} \leq C \prod_{j=1}^{n}\left(\alpha_{j}+n\right) !(2 a)^{-|\alpha|}
$$

for all $\alpha \in \mathbb{N}^{n}$. Then $F$ extends to $\mathbb{C}^{n}$ as an entire function which satisfies $|F(\zeta)| \leq$ $C e^{b|\Im \zeta|^{2}}$ for every $b>1 / 4 a$.

PROOF. For $b>1 / 4 a$,

$$
\begin{aligned}
|F(\xi+\eta)| & =\left|\sum_{\alpha} \frac{\partial^{\alpha} F(\xi)}{\alpha !} \eta^{\alpha}\right| \leq \sum_{\alpha}\left|\frac{\partial^{\alpha} F(\xi)}{\alpha !}\right||\eta|^{|\alpha|} \\
& =\sum_{\alpha} \frac{\left|\partial^{\alpha} F(\xi)\right|}{\alpha !^{1 / 2}}(2 b)^{-|\alpha| / 2} \frac{(2 b)^{|\alpha| / 2}}{\alpha !^{1 / 2}}|\eta|^{|\alpha|} \\
& \leq\left(\sum_{\alpha} \frac{\left|\partial^{\alpha} F(\xi)\right|^{2}}{\alpha !}(2 b)^{-|\alpha|}\right)^{1 / 2}\left(\sum_{\alpha} \frac{\left(|\eta|^{2} 2 b\right)^{|\alpha|}}{\alpha !}\right)^{1 / 2} \\
& \leq C\left(\sum_{\alpha} \frac{(\alpha+n) !}{\alpha !}\left(\frac{1}{4 a b}\right)^{|\alpha|}\right)^{1 / 2} e^{b|\eta|^{2}}=C(n, b) e^{b|\eta|^{2}} .
\end{aligned}
$$

This shows that $F$ can be extended as an entire function on $\mathbb{C}^{n}$ and it satisfies

$$
|F(\xi+i \eta)| \leq C(n, b) e^{b|\eta|^{2}} .
$$

Coming to the proof of the case $a b>1 / 4$, choose $b^{\prime}$ such that $b>b^{\prime}>1 / 4 a$. By Lemma 2.4, we have $|\hat{f}(\zeta)| \leq C e^{b^{\prime}|9 \xi|^{2}}$. Since $|\hat{f}(\xi)| \leq C e^{-b|\xi|^{2}}$ and $b^{\prime}<b$, we appeal to the following lemma to conclude that $\hat{f}=0$.

LEMMA 2.5. Let $F(\zeta)$ be an entire function on $\mathbb{C}^{n}$ that satisfies

$$
|F(\zeta)| \leq C e^{a|\Im \zeta|^{2}}, \quad|F(\xi)| \leq C e^{-b|\xi|^{2}}
$$

for $\zeta \in \mathbb{C}^{n}$ and $\xi \in \mathbb{R}^{n}$. Then $F=0$ whenever $a<b$ and $F(\zeta)=C e^{-a \zeta^{2}}$ for $a=b$. 
We consider the equality case. It is enough to prove it when $n=1$. Indeed, if we have the result in the one dimensional case then, by considering the function $F\left(\xi_{n}\right)=\hat{f}\left(\xi^{\prime}, \xi_{n}\right), \xi^{\prime}=\left(\xi_{1}, \ldots \xi_{n-1}\right)$, which satisfies the estimates

$$
\left|F\left(\xi_{n}\right)\right| \leq C\left(\xi^{\prime}\right) e^{-b \xi_{n}^{2}}\left|\partial^{k} F\left(\xi_{n}\right)\right|^{2} \leq C(k+n) !(2 a)^{-k},
$$

we obtain

$$
F\left(\xi_{n}\right)=C\left(\xi^{\prime}, \xi_{n}\right) e^{-b \xi_{n}^{2}}
$$

However, the function $C\left(\xi^{\prime}, \xi_{n}\right)$ satisfies the same estimates as $\hat{f}$ on $\mathbb{R}^{n-1}$. By induction we obtain $\hat{f}(\xi)=\phi(\xi) e^{-b|\xi|^{2}}$ with $\phi$ bounded. For the one-dimensional case using Lemma $2.4, \hat{f}$ can be extended to $\mathbb{C}$ as an entire function of order at most 2. Since $\hat{f}$ cannot decay on $\mathbb{R}$ faster than its order, its order is 2 . Since we have the estimate

$$
|\hat{f}(\zeta)| \leq C e^{b^{\prime}|\Im \zeta|^{2}} \quad \text { for all } b^{\prime}>1 / 4 a,
$$

its type is $1 / 4 a$. Now we apply the following result of Pfannschmidt [9] to the entire function $\hat{f}(\zeta)$.

THEOREM 2.6. Let $F$ be an entire function of one variable $\zeta$ of order $\rho$ ( $\rho$ integer) and type $b$. Let

$$
h(\theta)=\limsup _{r \rightarrow \infty} \frac{\log \left|F\left(r e^{i \theta}\right)\right|}{r^{\rho}}, \quad \theta \in[0, \infty)
$$

be its indicator and assume that $h(2 \pi j / \rho) \leq-b, j=0,1,2, \ldots, \rho-1$. Then $F(\zeta)=P(\zeta) e^{-b \zeta^{p}}$, where $P(\zeta)$ is an entire function at most of minimal type of order $\rho$.

The following remark is in order. In Theorem 1.3 , with $a b=1 / 4$, we concluded that $\hat{f}(\xi)=\phi(\xi) e^{-b|\xi|^{2}}$. It would be nice to say something about $f$ itself. As $\phi(\zeta)$ is an entire function, we have

$$
\phi(\xi)=\sum_{|\alpha| \leq N} a_{\alpha} \xi^{\alpha}+\phi_{N}(\xi)
$$

where $\left|\phi_{N}(\xi)\right| \leq c(1+|\xi|)^{N}$. This shows, in view of the inversion formula

$$
f(x)=(2 \pi)^{-n / 2} \int_{\mathbf{R}^{n}} e^{i x \cdot \xi} \phi(\xi) e^{-b|\xi|^{2}} d \xi,
$$

that $f$ can be written as

$$
f(x)=\left(\sum_{|\alpha| \leq N} c_{\alpha} x^{\alpha}\right) e^{-a|x|^{2}}+f_{N}(x),
$$

where $\hat{f}_{N}(\xi)=\phi_{N}(\xi) e^{-b|\xi|^{2}}$ 
Finally, it is easy to prove the equivalence between Theorem 1.3 (case $a b>1 / 4$ ) and Theorem 1.1 (case $p=2, q=\infty$ ). Assuming Theorem 1.1, consider Theorem 1.3 with $a b>1 / 4$. Choose $a^{\prime}<a$, but satisfying $a^{\prime} b>1 / 4$ and consider $f \phi_{a^{\prime}}$. Expanding the Gaussian, we have

$$
\begin{aligned}
\int_{\mathbb{R}^{n}}\left|f(x) \phi_{a^{\prime}}(x)\right|^{2} \cdot d x & =\sum_{k=0}^{\infty}\left(\int_{\mathbb{R}^{n}}|f(x)|^{2} \frac{\left(2 a^{\prime}\right)^{k}}{k !}|x|^{2 k} d x\right) \\
& =\sum_{k=0}^{\infty}\left(\sum_{|\alpha|=k} \frac{1}{\alpha !} \int_{\mathbb{R}^{n}}|f(x)|^{2} x^{2 \alpha} d x\right)\left(2 a^{\prime}\right)^{k} .
\end{aligned}
$$

Under the hypothesis on $\left\|\partial^{\alpha} \hat{f}\right\|_{2}^{2}$, we get

$$
\int_{\mathbb{R}^{n}}\left|f(x) \phi_{a^{\prime}}(x)\right|^{2} d x \leq C \sum_{k=0}^{\infty}\left(\sum_{|\alpha|=k} \frac{1}{\alpha !}\right)\left(\frac{a^{\prime}}{a}\right)^{k} \leq C \sum_{k=0}^{\infty} k^{n-1}\left(\frac{a^{\prime}}{a}\right)^{k}<\infty .
$$

Hence we can apply Theorem 1.1 to conclude that $f=0$.

The above calculation shows that $f \phi_{a}^{-1} \in L^{2}$ implies the estimates

$$
\left\|\partial^{\alpha} \hat{f}\right\|_{2}^{2} \leq C \alpha !(2 a)^{-|\alpha|}
$$

for every $\alpha \in \mathbb{N}^{n}$, and therefore Theorem 1.3 implies Theorem $1.1(p=2, q=\infty)$.

We also have the following implication: Theorem 2.2 implies Theorem $1.1(p=2$, $q=\infty$ ). In view of

$$
\partial^{\alpha} \hat{f}(\xi)=(2 \pi)^{-n / 2}(-i)^{|\alpha|} \int_{\mathbb{R}^{n}} e^{-i x \cdot \xi} f(x) x^{\alpha} d x,
$$

and by the Cauchy-Schwarz inequality, the assumption $f \phi_{a}^{-1} \in L^{2}$ gives

$$
\left|\partial^{\alpha} \hat{f}(\xi)\right|^{2} \leq C\left\|f \phi_{a}^{-1}\right\|_{2}^{2}\left\|x^{\alpha} \phi_{a}\right\|_{2}^{2} \leq C \alpha !(2 a)^{-|\alpha|} .
$$

Hence the hypothesis of Theorem 2.2 is satisfied.

\section{An uncertainty principle for operators}

In this section we prove Theorem 1.4 and Theorem 1.5. In order to prove these results we need to use several properties of the Weyl transform and special Hermite functions. We recall the relevant results, referring to $[13,11]$ for details.

The Weyl transform is closely related to the Fourier transform on the Heisenberg group $H^{n}$. Let $\pi_{\lambda}$ be the Schrödinger representations on $H^{n}$ with parameter $\lambda \in \mathbb{R} \backslash 0$. Explicitly $\pi_{\lambda}(z, t): L^{2}\left(\mathbb{R}^{n}\right) \rightarrow L^{2}\left(\mathbb{R}^{n}\right)$ is the unitary operator given by

$$
\pi_{\lambda}(z, t) \phi(\xi)=e^{i \lambda t} e^{i \lambda(x \cdot \xi+x \cdot y / 2)} \phi(\xi+y),
$$


where $\phi \in L^{2}\left(\mathbb{R}^{n}\right), \xi \in \mathbb{R}^{n}$ and $z=(x+i y)$. We define $\pi_{\lambda}(z)=\pi_{\lambda}(z, 0)$, so that $\pi_{\lambda}(z, t)=e^{i \lambda t} \pi_{\lambda}(z)$. For $f \in L^{1}\left(\mathbb{C}^{n}\right)$, its Weyl transform $W_{\lambda}(f)$ is the bounded operator on $L^{2}\left(\mathbb{R}^{n}\right)$ given by

$$
W_{\lambda}(f) \phi=\int_{\mathbb{C}^{n}} f(z) \pi_{\lambda}(z) \phi d z .
$$

It is clear that $\left\|W_{\lambda}(f)\right\| \leq\|f\|_{1}$. For $f \in L^{1} \cap L^{2}\left(\mathbb{C}^{n}\right), W_{\lambda}(f)$ is Hilbert-Schmidt and we have the Plancherel theorem

$$
\left\|W_{\lambda}(f)\right\|_{\mathrm{HS}}^{2}=(2 \pi)^{n}|\lambda|^{-n} \int_{\mathrm{C}^{n}}|f(z)|^{2} d z
$$

Thus $W_{\lambda}$ is an isometric isomorphism between $L^{2}\left(\mathbb{C}^{n}\right)$ and $\mathscr{S}_{2}$. For $f \in L^{1}\left(H^{n}\right)$, set

$$
f^{\lambda}(z)=\int_{-\infty}^{\infty} e^{i \lambda t} f(z, t) d t
$$

to be the inverse Fourier transform of $f$ in the $t$-variable. Then from the definition of $\hat{f}(\lambda)$ it follows that $\hat{f}(\lambda)=W_{\lambda}\left(f^{\lambda}\right)$. For $\lambda=1$, we define $W(z)=\pi_{1}(z)$ and $W(f)=W_{1}(f)$.

Given $f \in L^{1} \cap L^{2}\left(\mathbb{C}^{n}\right)$, we define the Fourier-Weyl transform of $f$ as the operator valued function $\tilde{f}(u, v)$ on $\mathbb{R}^{2 n}$ given by

$$
\tilde{f}(u, v)=W(u+i v) W(f) W(u+i v)^{*} .
$$

As $W(z)$ is a projective representation of $\mathbb{C}^{n}$, it is easily seen that

$$
\tilde{f}(u, v)=\int_{\mathbb{C}^{n}} e^{i(x \cdot v-y \cdot u)} f(x+i y) W(x+i y) d x d y .
$$

A simple calculation, using the definition, shows that $\delta_{j} W(f)=W\left(\bar{M}_{j} f\right)$ and $\bar{\delta}_{j} W(f)=W\left(M_{j} f\right)$, where $M_{j} f(z)=z_{j} f(z)$ and $\bar{M}_{j} f(z)=\bar{z}_{j} f(z)$. By iteration we get $\delta^{\alpha} W(f)=W\left(\bar{z}^{\alpha} f\right)$ and $\bar{\delta}^{\beta} W(f)=W\left(z^{\beta} f\right)$. Taking derivatives in $u, v$ and using these relations, we get

$$
\partial_{u}^{\alpha} \partial_{v}^{\beta} \tilde{f}(u, v)=2^{-(|\alpha|+|\beta|)} W(u+i v)(\delta+\bar{\delta})^{\beta}(\delta-\bar{\delta})^{\alpha} W(f) W(u+i v)^{*} .
$$

This identity shows that $\left\|\partial_{u}^{\alpha} \partial_{v}^{\beta} \tilde{f}(u, v)\right\|_{\mathrm{HS}}^{2} \leq C(\alpha+\beta) ! a^{|\alpha|+|\beta|}$ whenever we have $\left\|\delta^{\alpha} \bar{\delta}^{\beta} W(f)\right\|_{\mathrm{HS}}^{2} \leq C(\alpha+\beta) ! a^{|\alpha|+|\beta|}$. The special Hermite functions $\Phi_{\mu \nu}$ are defined as follows. Let $\Phi_{\mu}(x), x \in \mathbb{R}^{n}$ be the Hermite functions on $\mathbb{R}^{n}$. Then we define

$$
\Phi_{\mu \nu}(z)=(2 \pi)^{-n / 2}\left(W(z) \Phi_{\mu}, \Phi_{\nu}\right) .
$$


These functions form an orthonormal basis for $L^{2}\left(\mathbb{C}^{n}\right)$ and they are expressible in terms of Laguerre functions. For our purposes, we only require the formula

$$
\Phi_{0, \mu}(z)=(2 \pi)^{-n / 2}\left(\frac{1}{\mu !}\right)^{1 / 2}\left(-i \frac{z}{\sqrt{2}}\right)^{\mu} e^{-|z|^{2} / 4} .
$$

We refer to [11] for these and more on special Hermite functions.

With these preparations we embark on a proof of Theorem 1.4. For $T \in \mathscr{S}_{2}$, we define $\hat{T}(u, v)=W(u+i v) T W(u+i v)^{*}$, so that $\hat{T}(u, v)=\tilde{f}(u, v)$ if $T=W(f)$. Let $F$ be the function on $\mathbb{R}^{2 n}$ defined by

$$
F(u, v)=\left(\hat{T}(u, v)^{*} \hat{T}(u, v) \Phi_{0}, \Phi_{0}\right) .
$$

We claim that $F(u, v)$ satisfies the following two properties:

(i) $F(u, v)$ extends to $\mathbb{C}^{2 n}$ as an entire function, which satisfies the estimate $|F(\zeta)| \leq C e^{a_{1}|\operatorname{Im} \zeta|^{2}}$ for some $a_{1}<\left(1-e^{-4 b}\right) / 2$.

(ii) $F(u, v) \leq C e^{-\left(1-e^{-4 b}\right)\left(|u|^{2}+|v|^{2}\right) / 2}$.

Assuming this claim for a moment, we appeal to the following lemma.

LEMMA 3.1. Let $F(\zeta)$ be an entire function on $\mathbb{C}^{n}$ that satisfies $|F(\zeta)| \leq C e^{a|\operatorname{Im} \zeta|^{2}}$, $\zeta \in \mathbb{C}^{n}$ and $|F(\xi)| \leq C\left(1+|\xi|^{2}\right)^{m} e^{-b|\xi|^{2}}, \xi \in \mathbb{R}^{n}$. Then $F=0$ whenever $a<b$.

The lemma shows that $F=0$ whenever $a_{1}<\left(1-e^{-4 b}\right) / 2$. Since $a<2 \tanh 2 b$ we have coth $2 b<2 / a$ and so we can choose $b_{1}$ and $b_{2}$ such that coth $2 b<4 b_{1}<$ $4 b_{2}<2 / a$. This gives $b_{2}<1 / 2 a$ and $1+4 b_{1}>1+\operatorname{coth} 2 b=2 /\left(1-e^{-4 b}\right)$ or $2 /\left(1+4 b_{1}\right)<\left(1-e^{-4 b}\right)$. In our claim we can take $a_{1}=1 /\left(1+4 b_{1}\right)$ so that $F=0$.

Let $S=T^{*} T$. Then it follows that $\hat{S}(u, v)=\hat{T}(u, v)^{*} \hat{T}(u, v)$. If $S=W(f)$, $f \in L^{2}\left(\mathbb{C}^{n}\right)$, then we have

$$
\begin{aligned}
F(u, v) & =\left(W(u+i v) W(f) W(u+i v)^{*} \Phi_{0}, \Phi_{0}\right) \\
& =\int_{\mathbb{R}^{2 n}} e^{i(x \cdot v-y \cdot u)} f(x, y) e^{-\left(|x|^{2}+|y|^{2}\right) / 4} d x d y .
\end{aligned}
$$

Hence $F(u, v)=0$ implies $f(x, y)=0$, proving the theorem as $S=T^{*} T=W(f)=0$.

It remains to prove the claim with $a_{1}=1 /\left(1+4 b_{1}\right)$, where $b_{1}$ is chosen as above. As we indicated earlier, the estimates on $\delta^{\alpha} \bar{\delta}^{\beta} S$ give the estimates

$$
\left\|\partial_{u}^{\alpha} \partial_{v}^{\beta} \tilde{f}(u, v)\right\|_{\mathrm{HS}}^{2} \leq C(\alpha+\beta) ! a^{|\alpha|+|\beta|}
$$

for all $\alpha, \beta \in \mathbb{N}^{n}$ and hence $\left\|\partial_{u}^{\alpha} \partial_{v}^{\beta} F(u, v)\right\|_{2}^{2} \leq C(\alpha+\beta) ! a^{|\alpha|+|\beta|}$ for all $\alpha, \beta \in \mathbb{N}^{n}$.

Since $S=W(f)$, using the Plancherel theorem we have the estimates

$$
\int_{\mathbb{R}^{2 n}}\left|x^{\alpha} y^{\beta} f(x, y)\right|^{2} d x d y \leq C(\alpha+\beta) ! a^{(|\alpha|+|\beta|)} .
$$


We claim that $\int_{\mathbb{R}^{2 n}}|f(x, y)|^{2} e^{2 b_{2}\left(|x|^{2}+|y|^{2}\right)} d x d y<\infty$ for any $b_{2}<1 / 2 a$. To see this consider the series

$$
\sum_{k=0}^{\infty} \frac{1}{k !} \int_{\mathbb{R}^{2 n}}|f(x, y)|^{2}\left(2 b_{2}\right)^{k}\left(|x|^{2}+|y|^{2}\right)^{k} d x d y,
$$

which converges as long as $b_{2}<1 / 2 a$ as in Section 2 .

Now consider $F(u, v)$ given by

$$
F(u, v)=\int_{\mathbb{R}^{2 n}} f(x, y) e^{i(x \cdot v-y \cdot u)} e^{-\left(|x|^{2}+|y|^{2}\right) / 4} d x d y .
$$

By Hölder's inequality

$$
\left|\partial_{u}^{\alpha} \partial_{v}^{\beta} F(u, v)\right|^{2} \leq C \int_{\mathbb{R}^{2 n}}\left|x^{\alpha} y^{\beta}\right|^{2} e^{-2\left(b_{2}+1 / 4\right)\left(|x|^{2}+|y|^{2}\right)} d x d y,
$$

which gives the estimate $\left|\partial_{u}^{\alpha} \partial_{v}^{\beta} F(u, v)\right|^{2} \leq C(\alpha+\beta) !\left(2\left(b_{2}+1 / 4\right)\right)^{-(|\alpha|+|\beta|)}$. Appealing to Lemma 2.4 we see that $F(u, v)$ extends to an entire function of type $a_{2}$ where $a_{2}=1 /\left(1+4 b_{2}\right)$. Since $a_{2}<a_{1}$, we get $|F(\zeta)| \leq C e^{a_{1}|3 \zeta|^{2}}$ which proves claim (i).

The second claim is proved using the bound $T^{*} T \leq C e^{-2 b H}$. We have

$$
\begin{aligned}
F(u, v) & =\left(W(u+i v) T^{*} T W(u+i v)^{*} \Phi_{0}, \Phi_{0}\right) \\
& \leq C\left(W(u+i v) e^{-2 b H} W(u+i v)^{*} \Phi_{0}, \Phi_{0}\right) .
\end{aligned}
$$

We now expand $W(u+i v)^{*} \Phi_{0}=W(u-i v) \Phi_{0}$ in terms of $\Phi_{\mu}$. This yields

$$
W(u+i v)^{*} \Phi_{0}=(2 \pi)^{n / 2} \sum \Phi_{0, \mu}(u-i v) \Phi_{\mu} .
$$

Since $e^{-2 b H} \Phi_{\mu}=e^{-2 b(2|\mu|+n)} \Phi_{\mu}$, we have, using Parseval's formula for Hermite expansions, $F(u, v) \leq C \sum e^{-2 b(2|\mu|+n)}\left|\Phi_{0, \mu}(u-i v)\right|^{2}$. Now using the explicit formula for $\Phi_{0, \mu}$ we get

$$
F(u, v) \leq C \sum e^{-2 b(2|\mu|+n)} \frac{1}{\mu !}\left(\frac{1}{2}\left(|u|^{2}+|v|^{2}\right)\right)^{|\mu|} e^{-\left(|u|^{2}+|v|^{2}\right) / 2}
$$

which gives $F(u, v) \leq C e^{-\left(1-e^{-4 b}\right)\left(|u|^{2}+|v|^{2}\right) / 2}$ as desired. This completes the proof of Theorem 1.4.

Let $f$ be a function in $L^{2}\left(\mathbb{C}^{n}\right)$ that is invariant under the action on $\mathbb{T}^{n}$. Then $f$ is called polyradial and its expansion is given by $f(z)=\sum_{\mu}\left(f, \Phi_{\mu \mu}\right) \Phi_{\mu \mu}(z)$. Let $d$ be a function on $\mathbb{N}$. For each $j \in \mathbb{N}$, we define the difference operators $\Delta_{j}^{+}$and $\Delta_{j}^{-}$by

$$
\left(\Delta_{j}^{+} d\right)(\mu)=d\left(\mu+e_{j}\right)-d(\mu), \quad\left(\Delta_{j}^{-} d\right)(\mu)=d(\mu)-d\left(\mu-e_{j}\right) .
$$


For multi-indices $\alpha, \beta \in \mathbb{N}$, we define

$$
\Delta_{+}^{\alpha}=\left(\Delta_{1}^{+}\right)^{\alpha_{1}}\left(\Delta_{2}^{+}\right)^{\alpha_{2}} \cdots\left(\Delta_{n}^{+}\right)^{\alpha_{n}}, \quad \Delta_{-}^{\beta}=\left(\Delta_{1}^{-}\right)^{\beta_{1}}\left(\Delta_{2}^{-}\right)^{\beta_{2}} \cdots\left(\Delta_{n}^{-}\right)^{\beta_{n}} .
$$

With these notations we prove the following corollary of Theorem 1.4 , which can be considered as the Cowling-Price theorem for the Laguerre expansion of polyradial functions.

COROLlARY 3.2. Let $f$ be a polyradial function which is in $L^{2}\left(\mathbb{C}^{n}\right), C(\mu)=$ $\left(f, \Phi_{\mu \mu}\right)$ and $d(\mu)=|C(\mu)|^{2}, \mu \in \mathbb{N}^{n}$. If $C(\mu)$ satisfy the following conditions:

(i) $|C(\mu)| \leq C e^{-b(2|\mu|+n)}$,

(ii) $\sum_{\mu} \frac{(\mu \mp \alpha) !}{(\mu-\beta) !}\left|\left(\Delta_{-}^{\beta} \Delta_{+}^{\alpha} d\right)(\mu)\right|^{2} \leq C(\alpha+\beta) ! 2^{-(|\alpha|+|\beta|)} a^{(|\alpha|+|\beta|)}$,

where $a, b>0$, then $f=0$ whenever $a<2 \tanh 2 b$.

PROOF. Since $f$ is polyradial, $W(f) \phi=\sum_{\mu}\left(f, \Phi_{\mu \mu}\right)\left(\phi, \Phi_{\mu}\right) \Phi_{\mu}$. Using the formulae

$$
A_{j} \Phi_{\mu}=\left(2 \mu_{j}+2\right)^{1 / 2} \Phi_{\mu+e_{j}}, \quad A_{j}^{*} \Phi_{\mu}=\left(2 \mu_{j}\right)^{1 / 2} \Phi_{\mu-e_{j}}
$$

it is easy to see that

$$
\left(\delta^{\alpha} \bar{\delta}^{\beta} W(f)^{*} W(f)\right) \Phi_{\mu}=(-1)^{|\alpha|+|\beta|} 2^{(|\alpha|+|\beta|) / 2}\left(\frac{(\mu+\alpha) !}{(\mu-\beta) !}\right)^{1 / 2}\left(\Delta_{-}^{\beta} \Delta_{+}^{\alpha} d\right)(\mu) \Phi_{\mu+\alpha-\beta}
$$

Then, using the above conditions, we get

$$
W(f)^{*} W(f) \leq C e^{-2 b H}, \quad\left\|\delta^{\alpha} \bar{\delta}^{\beta}\left(W(f)^{*} W(f)\right)\right\|_{\mathrm{HS}}^{2} \leq C(\alpha+\beta) ! a^{|\alpha|+\mid \beta !} .
$$

So by Theorem 1.4, $W(f)=0$ for $a<2 \tanh 2 b$ and hence $f=0$.

\section{Cowling-Price theorem for Heisenberg groups}

In this section we prove Theorem 1.2, which is an analogue of the Cowling-Price theorem for the Heisenberg group $H^{n}$. Before going to the proof of Theorem 1.2, let us prove the following theorem which can be considered as the Cowling-Price theorem for Weyl transforms. Theorem 1.5 will be proved as a special case $\lambda=1$.

THEOREM 4.1. Let $f e^{a|z|^{2}} \in L^{p}\left(\mathbb{C}^{n}\right)$ and $W_{\lambda}(f) e^{b H(\lambda)} \in \mathscr{S}_{q}$, where $1 \leq p, q \leq \infty$. Then $f=0$ almost everywhere whenever $(a \tanh b \lambda) / \lambda \geq 1 / 4$ and $\min (p, q)<\infty$. If $p=q=\infty$, then $f=0$ for $(a \tanh b \lambda) / \lambda>1 / 4$ and $f(z)=C e^{-a|z|^{2}}$ for $(a \tanh b \lambda) / \lambda=1 / 4$. 
PROOF. Let $\mathbb{T}^{n}$ denote the subgroup of the unitary group $U(n)$ consisting of diagonal matrices. Then each element of $\mathbb{T}^{n}$ can be written in the form $e^{i \theta}=\left(e^{i \theta_{1}}, \ldots, e^{i \theta_{n}}\right)$ so that $\mathbb{T}^{n}$ can be identified with the $n$-torus. The action of the $n$-torus on $\mathbb{C}^{n}$ is given by $e^{i \theta} \cdot z=\left(e^{i \theta_{1}} z_{1}, e^{i \theta_{2}} z_{2}, \ldots, e^{i \theta_{n}} z_{n}\right)$. Let $m$ be an $n$-tuple of integers. We say $g$ is $m$-homogeneous if $g\left(e^{i \theta} . z\right)=e^{i m . \theta} g(z)$ for all $\theta$. It is easy to see that when $g$ is $m$ homogeneous and $h$ is $k$-homogeneous, then $(g, h)=0$ unless $m=k$. Since $f e^{a|z|^{2}}$ is in $L^{p}\left(\mathbb{C}^{n}\right)$, it follows that $f_{m} e^{a|z|^{2}} \in L^{p}\left(\mathbb{C}^{n}\right)$, where $f_{m}(z)=\int_{\mathbb{T}^{n}} f\left(e^{i \theta} \cdot z\right) e^{-i m \cdot \theta} d \theta$. Hence

$$
\begin{aligned}
W_{\lambda}\left(f_{m}\right) & =\int_{\mathbb{C}^{n}} \int_{\mathbb{T}^{n}} f\left(e^{i \theta} \cdot z\right) \pi_{\lambda}(z) e^{-i m \cdot \theta} d \theta d z \\
& =\int_{\mathbb{C}^{n}} \int_{\mathbb{T}^{n}} f\left(e^{i \theta} \cdot z\right) \pi_{\lambda}\left(e^{-i \theta} \cdot z\right) e^{-i m \cdot \theta} d \theta d z \\
& =\int_{\mathbb{J}^{n}} \mu_{\lambda}(\theta)^{*} W_{\lambda}(f) \mu_{\lambda}(\theta) e^{-i m \cdot \theta} d \theta,
\end{aligned}
$$

where $\mu_{\lambda}(\theta)$ is a unitary operator on $L^{2}\left(\mathbb{R}^{n}\right)$ such that

$$
\pi_{\lambda}\left(e^{i \theta} . z\right)=\mu_{\lambda}(\theta)^{*} \pi_{\lambda}(z) \mu_{\lambda}(\theta) .
$$

We refer to [13] for several properties of $\mu_{\lambda}(\theta)$. Using the above expression for $W_{\lambda}\left(f_{m}\right)$, we get

$$
\begin{aligned}
\left\|W_{\lambda}\left(f_{m}\right) \Phi_{\alpha}\right\|_{2} & \leq \int_{\mathbb{T}^{n}}\left\|\mu_{\lambda}(\theta)^{*} W_{\lambda}(f) \mu_{\lambda}(\theta) \Phi_{\alpha}\right\|_{2} d \theta \\
& =\int_{\mathbf{T}^{n}}\left\|W_{\lambda}(f) \mu_{\lambda}(\theta) \Phi_{\alpha}^{\lambda}\right\|_{2} d \theta .
\end{aligned}
$$

Now $E_{k}^{\lambda}=\operatorname{span}\left\{\Phi_{\alpha}^{\lambda}:|\alpha|=k\right\}$ is invariant under the action of $\mu_{\lambda}(\theta)$. Therefore,

$$
\mu_{\lambda}(\theta) \Phi_{\alpha}^{\lambda}=\sum_{|\beta|=k} C_{\alpha \beta}^{\lambda}(\theta) \Phi_{\beta}^{\lambda}
$$

for all $\theta \in \mathbb{T}^{n}$. As $\mu_{\lambda}(\theta)$ is a unitary operator,

$$
\sum_{|\beta|=k}\left|C_{\alpha \beta}^{\lambda}(\theta)\right|^{2}=1
$$

for all $\theta \in \mathbb{T}^{n}$. Since every member of $\mathscr{S}_{q}$ is bounded and $W_{\lambda}(f) e^{b H(\lambda)} \in \mathscr{S}_{q}$, there exists $C>0$ such that $\left\|W_{\lambda}(f) \Phi_{\alpha}^{\lambda}\right\|_{2} \leq C e^{-b(2|\alpha|+n)|\lambda|}$ for all $\alpha \in \mathbb{N}^{n}$. Now using these estimates on $W_{\lambda}(f)$ and applying the Cauchy-Schwarz inequality we get

$$
\left\|W_{\lambda}(f) \mu_{\lambda}(\theta) \Phi_{\alpha}^{\lambda}\right\|_{2}^{2} \leq C \frac{(k+n-1) !}{k !(n-1) !} e^{-2 b(2 k+n)|\lambda|}
$$


for all $\Phi_{\alpha}^{\lambda}$ with $|\alpha|=k$. So, for all $\alpha$ with $|\alpha|=k$,

$$
\begin{aligned}
\left|\left(f_{m}, \Phi_{\alpha, \alpha+m}^{\lambda}\right)\right| & =\left|\left(W_{\lambda}\left(f_{m}\right) \Phi_{\alpha}^{\lambda}, \Phi_{\alpha+m}^{\lambda}\right)\right| \\
& \leq\left\|W_{\lambda}\left(f_{m}\right) \Phi_{\alpha}^{\lambda}\right\|_{2} \\
& \leq C_{n} \sqrt{\frac{\Gamma(k+n)}{\Gamma(k+1)}} e^{-b(2 k+n)|\lambda|} \\
& \leq C_{n} k^{n} e^{-b(2 k+n)|\lambda|} \\
& \leq C_{n} \prod_{i=1}^{n}\left(\left(2 \alpha_{i}+1\right)^{n} e^{-b\left(2 \alpha_{i}+1\right)|\lambda|}\right) .
\end{aligned}
$$

Let $\mathscr{F}_{\lambda} f$ stand for the symplectic Fourier transform of a function $f$ in $\mathbb{C}^{n}$ defined by

$$
\mathscr{F}_{\lambda} f(z)=(2 \pi)^{-n} \int_{\mathbf{C}^{n}} f(z-w) e^{i \lambda \operatorname{Im}(z \cdot \bar{w}) / 2} d w .
$$

$\mathscr{F}_{\lambda}$ is related to the ordinary Fourier transform by $\mathscr{F}_{\lambda} f(z)=\hat{f}(-i \lambda z / 2)$. As $f_{m}$ is $m$-homogeneous and $\Phi_{\alpha \beta}$ is $(\beta-\alpha)$-homogeneous expanding $f_{m}$, we get

$$
f_{m}(z)=\sum_{\beta}\left(f_{m}, \Phi_{\beta, \beta+m}\right) \Phi_{\beta, \beta+m} .
$$

As $\mathscr{F}_{\lambda} \Phi_{\alpha, \alpha+m}^{\lambda}=(-1)^{|m|} \Phi_{\alpha, \alpha+m}^{\lambda}$,

$$
\mathscr{F}_{\lambda} f_{m}(z)=\sum_{m}(-1)^{|m|}\left(f_{m}, \Phi_{\alpha, \alpha+m}^{\lambda}\right) \Phi_{\alpha, \alpha+m}^{\lambda}
$$

For a proof of this we refer to [13]. The functions $\Phi_{\alpha, \alpha+m}^{\lambda}$ are expressible as products of one-dimensional Laguerre functions, and so without loss of generality we can assume that $n=1$ and $m$ is an integer. Explicit formula for $\Phi_{k, k+m}^{\lambda}$ is given as follows

$$
\Phi_{k, k+m}^{\lambda}(z)= \begin{cases}\lambda^{1 / 2} \Phi_{k, k+m}(\sqrt{\lambda} z) & \text { for } \lambda>0, \\ |\lambda|^{1 / 2} \Phi_{k, k+m}(\sqrt{|\lambda|} z) & \text { for } \lambda<0,\end{cases}
$$

where

$$
\Phi_{k, k+m}(z)=(2 \pi)^{-1 / 2}\left(\frac{-i}{\sqrt{2}}\right)^{m}\left(\frac{\Gamma(k+1)}{\Gamma(k+m+1)}\right)^{1 / 2} z^{m} \phi_{k}^{m}(z)
$$

and $\phi_{k}^{m}(z)$ are the Laguerre functions on $\mathbb{C}^{n}$ defined by

$$
\phi_{k}^{m}(z)=L_{k}^{m}\left(|z|^{2} / 2\right) e^{-1 / 4|z|^{2}} .
$$


Therefore,

$$
\begin{aligned}
\left|\mathscr{F}_{\lambda} f_{m}(z)\right| & \leq C_{n}(\lambda) \sum_{k=0}^{\infty}(2 k+1)|\lambda| e^{-b(2 k+1)|\lambda|}\left|\Phi_{k, k+m}^{\lambda}(z)\right| \\
& \leq C_{n, m}(\lambda)|z|^{m} \sum_{k=0}^{\infty} \frac{(2 k+m+1)|\lambda|}{e^{b(2 k+m+1)|\lambda|}}\left(\frac{\Gamma(k+1)}{\Gamma(k+m+1)}\right)^{1 / 2}\left|\phi_{k, \lambda}^{m}(z)\right| \\
& \leq C_{n, m}(\lambda)|z|^{m}\left(\sum_{k=0}^{\infty} \frac{((2 k+m+1)|\lambda|)^{4}}{e^{2 b(2 k+m+1)|\lambda|}}\left(\frac{\Gamma(k+1)}{\Gamma(k+m+1)}\right)\left(\phi_{k, \lambda}^{m}(z)\right)^{2}\right)^{1 / 2} \\
& \leq C_{n, m}(\lambda)\left(1+|\lambda||z|^{2}\right)^{l} e^{-(\lambda \tanh b \lambda)|z|^{2} / 4}
\end{aligned}
$$

for some positive integer $l>0$ (see [15, page 93]).

Using the relation $\mathscr{F}_{\lambda} f_{m}(z)=\hat{f}_{m}(-i \lambda z / 2)$, we have

$$
\left|\hat{f}_{m}(z)\right| \leq C_{n, m}(\lambda)\left(1+|z|^{2}\right)^{l} e^{-(\tanh b \lambda)|z|^{2} / \lambda} .
$$

So by the Cowling-Price theorem for Fourier transforms we conclude the following:

Case 1. If $p<\infty, q \leq \infty$, then for $(a \tanh b \lambda) / \lambda \geq 1 / 4, f_{m}=0$ for all $m$ and hence $f=0$ almost everywhere.

Case 2. If $p=\infty, q<\infty$, then for $(a \tanh b \lambda) / \lambda>1 / 4, f_{m}=0$ and for $(a \tanh b \lambda) / \lambda=1 / 4, f_{m}(z)=C_{m} e^{-a|z|^{2}}$ for all $m$. Since $f_{m}$ is $m$-homogeneous, $C_{m}=0$, except $m=0$ and hence $f(z)=f_{0}(z)=C e^{-a|z|^{2}}$ which yields $W_{\lambda}(f)=$ $C e^{-b H(\lambda)}$. However, almost everywhere, this is not compatible with the condition $W_{\lambda}(f) e^{b H(\lambda)} \in \mathscr{S}_{q}$ and hence $f=0$.

Case 3. If $p=q=\infty$, then $f_{m}=0$ for all $m$ whenever $(a \tanh b \lambda) / \lambda>1 / 4$, and for $(a \tanh b \lambda) / \lambda=1 / 4$, arguing as before, $f(z)=f_{0}(z)=C e^{-a|z|^{2}}$.

The special Hermite operator $L_{\lambda}$ is defined by the relation

$$
\mathscr{L}\left(e^{i \lambda t} f(z)\right)=e^{i \lambda t} L_{\lambda} f(z),
$$

where $\mathscr{L}$ is the sublaplacian on the Heisenberg group. This operator plays an important role in harmonic analysis and we refer to $[15,11]$ for details.

CoROLlaRY 4.2. If $f\left(q_{a}^{\lambda}\right)^{-1} \in L^{p}\left(\mathbb{C}^{n}\right)$ and $W_{\lambda}(f) e^{b H(\lambda)} \in \mathscr{S}_{q}, 1 \leq p, q \leq \infty$, where $q_{a}^{\lambda}$ is the heat kernel corresponding to the special Hermite operator, then $f=0$ almost everywhere whenever $a \leq b$ and $\min (p, q)<\infty$. If $p=q=\infty$, then $f=0$ for $a<b$ and $f(z)=C(\lambda) q_{a}^{\lambda}(z)$ for $a=b$.

PROOF. The explicit formula for the heat kernel $q_{a}^{\lambda}$ corresponding to the special Hermite operator is $q_{a}^{\lambda}(z)=(4 \pi)^{-n}(\lambda / \sinh \lambda a)^{n} e^{-\lambda(\operatorname{coth} a \lambda)|z|^{2} / 4}$. Since $a \leq b$ and $\tanh (\cdot)$ is an increasing function, $\tanh a|\lambda| \leq \tanh b|\lambda|$. So we get the desired result applying Theorem 4.1 . 
To prove Theorem 1.2, let $g(z, t)=q_{a}^{-1}(z, t) f(z, t)$. Then $g \in L^{p}\left(H^{n}\right)$. Using the estimate $\left|q_{a}(z, t)\right| \leq C a^{-n-1} e^{-A\left(\left.z\right|^{2}+|t|\right) / a}$ for some $C, A>0$ and the condition $g \in L^{p}\left(H^{n}\right)$, it is easy to see that

$$
f^{\lambda}(z)=\int_{-\infty}^{\infty} e^{i \lambda t} q_{a}(z, t) g(z, t) d t
$$

can be extended as a holomorphic function in the strip $|\Im \lambda|<A / a$ of the complex plane.

For $p=\infty$,

$$
\left|f^{\lambda}(z)\right| \leq\|g(z, \cdot)\|_{\infty} \int_{-\infty}^{\infty}|q(z, t)| d t \leq\|g(z, \cdot)\|_{\infty} e^{-|z|^{2} / 4 a} .
$$

For $1 \leq p \leq 2$,

$$
\begin{aligned}
\left|f^{\lambda}(z)\right| & \leq \int_{-\infty}^{\infty}\left|q_{a}(z, t)\right||g(z, t)| d t \\
& \leq\left(\int_{-\infty}^{\infty}\left|q_{a}(z, t)\right|^{p^{\prime}} d t\right)^{1 / p^{\prime}}\left(\int_{-\infty}^{\infty}|g(z, t)|^{p} d t\right)^{1 / p} \\
& \leq\left(\int_{-\infty}^{\infty}\left|q_{a}^{\lambda}(z)\right|^{p} d \lambda\right)^{1 / p}\left(\int_{-\infty}^{\infty}|g(z, t)|^{p} d t\right)^{1 / p} \\
& \leq(4 \pi)^{-n}\left(\int_{-\infty}^{\infty}\left(\frac{\lambda}{\sinh a \lambda}\right)^{p n} e^{-p \lambda(\operatorname{coth} a \lambda)|z|^{2} / 4} d \lambda\right)^{1 / p}\left(\int_{-\infty}^{\infty}|g(z, t)|^{p} d t\right)^{1 / p} \\
& \leq C e^{-|z|^{2} / 4 a}\left(\int_{-\infty}^{\infty}|g(z, t)|^{p} d t\right)^{1 / p} .
\end{aligned}
$$

When $2<p<\infty, 1<p^{\prime}<2$, we write $1 / p^{\prime}=v / 1+(1-v) / 2$ for some $0<v<1$. Since $\left\|q_{a}(z, \cdot)\right\|_{1} \leq e^{-1 / 4 a|z|^{2}}$ and $\left\|q_{a}(z, \cdot)\right\|_{2} \leq e^{-1 / 4 a|z|^{2}}$, applying Hölder's inequality with the pair of conjugate exponents $1 /\left(\nu p^{\prime}\right)$ and $2 /(1-\nu) p^{\prime}$, we get

$$
\begin{aligned}
\int_{-\infty}^{\infty}\left|q_{a}(z, t)\right|^{p^{\prime}} d t & =\int_{-\infty}^{\infty}\left|q_{a}(z, t)\right|^{v p^{\prime}}\left|q_{a}(z, t)\right|^{(1-v) p^{\prime}} d t \\
& \leq\left\|q_{a}(z, \cdot)\right\|_{1}^{v p^{\prime}}\left\|q_{a}(z, \cdot)\right\|_{2}^{(1-v) p^{\prime}},
\end{aligned}
$$

which gives $\left\|q_{a}(\cdot, t)\right\|_{p^{\prime}} \leq e^{-|z|^{2} / 4 a}$. Therefore, $f^{\lambda} e^{|z|^{2} / 4 a} \in L^{p}\left(\mathbb{C}^{n}\right)$ for $1 \leq p \leq \infty$. Also we have that $W_{\lambda}\left(f^{\lambda}\right) e^{b H(\lambda)}=\hat{f}(\lambda) e^{b H(\lambda)}$ is a bounded operator on $L^{2}\left(\mathbb{R}^{n}\right)$ for every $\lambda \in \mathbb{R} \backslash 0$.

Since $a<b$, we can choose $\delta>0$ such that $a\left(e^{b \delta}+\dot{e}^{-b \delta}\right)<2 b$. Now, for $0<\lambda<\delta$,

$$
\tanh b \lambda=\frac{e^{b \lambda}-e^{-b \lambda}}{e^{b \lambda}+e^{-b \lambda}}>\frac{2 b \lambda}{e^{b \lambda}+e^{-b \lambda}}>\frac{2 b \lambda}{e^{b \delta}+e^{-b \delta}}>a \lambda
$$


Using Theorem 4.1, we conclude that $f^{\lambda}=0$ for $0<\lambda<\delta$. Hence $f^{\lambda}=0$ for all $\lambda$ as $f^{\lambda}$ can be extended to a holomorphic function in the strip $|\Im \lambda|<A / a$. So $f=0$ almost everywhere.

REMARK 4.3. We can assume $\hat{f}(\lambda) e^{b H(\lambda)} \in \mathscr{S}_{q}, 1 \leq q \leq \infty$ in Theorem 1.2 as every member in $\mathscr{S}_{q}$ is also a bounded operator.

Acknowledgement The authors wish to thank the referee for his comments and suggestions which helped to improve the exposition of the paper.

\section{References}

[1] F. Astengo, M. Cowling, B. Di Blasio and M. Sundari, 'Hardy's uncertainty principle on certain Lie groups', J. London Math. Soc. 62 (2000), 461-472.

[2] S. C. Bagchi and S. K. Ray, 'Uncertainty principles like Hardy's theorem on some Lie groups', J. Aust. Math. Soc. 65 (1999), 289-302.

[3] A. Baklouti and N. B. Salah, 'The $L^{p}-L^{q}$ version of Hardy's theorem on nilpotent Lie groups', Forum Math. 18 (2006), 245-262.

[4] A. Bonami, B. Demange and P. Jaming, 'Hermite functions and uncertainty principles for the Fourier and the windowed Fourier transforms', Rev. Math. Iberoamericano 19 (2003), 23-55.

[5] M. Cowling and J. Price, 'Generalisations of Heisenberg's inequality', in: Harmonic analysis (eds. G. Mauceri, F. Ricci and G. Weiss), Lecture Notes in Math. 992 (Springer, Berlin, 1983).

[6] G. H. Hardy, 'A theorem concerning Fourier transforms', J. London Math. Soc. 8 (1933), 227-231.

[7] E. Kaniuth and A. Kumar, 'Hardy's theorem for simply connected nilpotent Lie groups', Proc. Cambridge Philos. Soc. 131 (2001), 487-494.

[8] G. Mauceri, 'The Weyl transform and bounded operators on $L^{p}\left(\mathbb{R}^{n}\right)$ ', J. Funct. Anal. 39 (1980), 408-429.

[9] C. Pfannschmidt, 'A generalization of the theorem of Hardy: A most general version of the uncertainty principle for Fourier integrals', Math. Nachr. 182 (1996), 317-327.

[10] A. Sitaram, M. Sundari and S. Thangavelu, 'Uncertainty principles on certain Lie groups', Proc. Indian Acad. Sci. 105 (1995), 135-151.

[11] S. Thangavelu, Lectures on Hermite and Laguerre expansions, Math. Notes. 42 (Princeton University Press, Princeton, NJ, 1993).

[12] — 'On Paley-Wiener theorems for the Heisenberg group', J. Funct. Anal. 115 (1993), 24-44.

[13] - Harmonic analysis on the Heisenberg group, Progr. Math. 159 (Birkhäuser, Boston, 1998).

[14] —..., 'Hardy's theorem on the Heisenberg group revisited', Math. Z. 242 (2002), 761-779.

[15] _ An introduction to the uncertainty principle: Hardy's theorem on Lie groups, Progr. Math. 217 (Birkhäuser, Boston, 2004).

Stat.-Math. Division

Indian Statistical Institute

203 B.T. Road

Kolkata 700108

India
Department of Mathematics Indian Institute of Science

Bangalore 560012

India

e-mail:veluma@math.iisc.ernet.in

e-mail: sanjuparui@gmai.com 